\title{
ON COMMUTATIVITY OF C*-ALGEBRAS
}

\author{
by C.-S. LIN
}

(Received 7 October, 1985)

1. Two numerical characterizations of commutativity for $\mathrm{C}^{*}$-algebra $\mathscr{A}$ (acting on the Hilbert space $H$ ) were given in [1]; one used the norms of self-adjoint operators in $\mathscr{A}$ (Theorem 2), and the other the numerical index of $\mathscr{A}$ (Theorem 3). In both cases the proofs were based on the result of Kaplansky which states that if the only nilpotent operator in $\mathscr{A}$ is 0 , then $\mathscr{A}$ is commutative ([2] 2.12.21, p. 68). Of course the converse also holds.

We shall apply in this note both Kaplansky's result and Holbrook's operator radii [3] to give two types of characterizations; one is by means of operator radii, and the other in terms of $C_{p}$-classes of operators in $\mathscr{A}$. These also enable us to generalize Theorem 2 and 3 in [1]. Finally, a particular case of our Theorem 5 shows that $\mathscr{A}$ is commutative if and only if every $T \in \mathscr{A}$ satisfies the first order growth condition $\left(G_{1}\right)$.

2. First we need some notation, definitions and well known results. Let $T \in \mathscr{A}$. We recall that $T$ is in the class $C_{p}(p>0)$, operators having unitary $p$-dilation, if

$$
(p h, h)-2 \operatorname{Re}(z(p-1) T h, h)+|z|^{2}((p-2) T h, T h) \geqslant 0
$$

holds for all $h \in H$ and $|z| \leqslant 1$ ([4 p. 45]). Since the inequality can be rewritten as $\operatorname{Re}\left(p-2 z(p-1) T+|z|^{2}(p-2) T^{*} T\right) \geqslant 0$, it follows easily that $T \in C_{p}$ if and only if for all $|z| \leqslant 1$ we have

$$
(p-2)(I-z T)^{*}(I-z T)+(I-z T)+(I-z T)^{*} \geqslant 0 .
$$

We need the following properties from [3].

(1) $T \in C_{p}$ if and only if $w_{p}(T) \leqslant 1$, where

$$
w_{p}(T)=\inf \left\{u: u>0, T / u \in C_{p}\right\},
$$

the operator radius of $T$.

(2) $w_{1}(T)=\|T\|$, and $w_{2}(T)$ is the numerical radius of $T$.

(3) $w_{p}(u T)=|u| w_{p}(T)$, and $w_{p}(T) \geqslant\|T\| / p$.

(4) $w_{p}($.$) is a norm on \mathscr{A}$ whenever $0<p \leqslant 2$.

Theorem 1 (Theorem 4.4, 4.5 and 5.5 [3]). Let $T \in \mathscr{A}$,

(1) If $0<p<p^{\prime}$, then $w_{p},(T) \leqslant w_{p}(T)$ and $w_{p}(T) \leqslant\left(\frac{2 p^{\prime}}{p}-1\right) w_{p},(T)$.

(2) If $\|T\|=1$ and $T^{2}=0$, then $w_{p}(T)=1 / p$ for every $p>0$.

Glasgow Math. J. 29 (1987) 93-97. 
(3) If $T$ is normaloid, that is $\|T\|$ is the spectral radius of $T$, then

$$
w_{p}(T)= \begin{cases}\left(\frac{2}{p}-1\right)\|T\| & \text { if } 0<p<1, \\ \|T\| & \text { if } p \geqslant 1 .\end{cases}
$$

Note that a normal operator is normaloid.

3. Now we are ready to give characterizations.

THEOREM 2. For $p>0$ and $p \neq 1, \mathscr{A}$ is commutative if and only if

$$
w_{p}(T)= \begin{cases}\left(\frac{2}{p}-1\right)\|T\| & \text { if } 0<p<1, \\ \|T\| & \text { if } p>1,\end{cases}
$$

for every $T \in \mathscr{A}$.

Proof. $(\Rightarrow)$. The commutativity implies that every $T \in \mathscr{A}$ is normal and so we may apply (3) in Theorem 1.

$(\Leftarrow)$. If $\mathscr{A}$ is not commutative, then there exists a $T \in \mathscr{A}, T \neq 0$ and $T^{2}=0$ such that $w_{p}(T)=\|T\| / p, p>0$ by (2) in Theorem 1 . Hence the equality in the statement does not hold.

Corollary 1. For $p>0$ and $p \neq 1, \mathscr{A}$ is not commutative if and only if $w_{p}(T)=\|T\| / p$ for some $\mathrm{T} \in \mathscr{A}$.

From the well known results in section two we see that

$$
\|T\| / p \leqslant w_{p}(T) \leqslant\left(\frac{2-p}{p}\right)\|T\|
$$

holds for $0<p<1$, and $\|T\| / p \leqslant w_{p}(T) \leqslant\|T\|$ for $p \geqslant 1$. Let us define

$$
n_{p}(\mathscr{A})=\inf \left\{w_{p}(T): T \in \mathscr{A},\|T\|=1\right\} .
$$

Clearly, $1 / p \leqslant n_{p}(\mathscr{A}) \leqslant \frac{2-p}{p}$ for $0<p<1$, and $1 / p \leqslant n_{p}(\mathscr{A}) \leqslant 1$ for $p \geqslant 1$. Note that $n_{2}(\mathscr{A})$ is called the numerical index of $\mathscr{A}$.

Corollary 2. (Theorem 3 [1] when $p=2$ ). (1) For $p>1, \mathscr{A}$ is commutative or not commutative according to $n_{p}(\mathscr{A})$ is 1 or $1 / p$.

(2) For $0<p<1, \mathscr{A}$ is commutative or not commutative according to $n_{p}(\mathscr{A})$ is $\frac{2-p}{p}$ or $1 / p$.

Proof. This is a simple consequence of Theorem 2 and Corollary 1. 
THEOREM 3. The following statements are equivalent.

(1) $\mathscr{A}$ is commutative.

(2) $c T \notin C_{p}$ for all $p \geqslant 1$, all $T$ with $0 \neq T \in \mathscr{A}$ and any $c>\|T\|^{-1}$.

(3) $\left(\frac{c p}{2-p}\right) T \notin C_{p}$ for all $p$ with $0<p<1$, all $T$ with $0 \neq T \in \mathscr{A}$ and any $c>\|T\|^{-1}$.

Proof. $(1) \Rightarrow(2)$. Since $c T$ is normal, $w_{p}(c T)=c\|T\|>1$ for every $p \geqslant 1$ by (3) in Theorem 1. Hence, $c T \notin C_{p}$.

$(2) \Rightarrow(1)$. If $\mathscr{A}$ is not commutative, then $w_{p}(c T)=c\|T\| / p$ for some $T \in \mathscr{A}, T \neq 0$ and $T^{2}=0$. We may select a suitable $p>1$ so that $p \geqslant c\|T\|$. This implies that $c T \in C_{p}$.

(1) $\Rightarrow$ (3). $w_{p}\left(\frac{c p}{2-p} T\right)=\left(\frac{c p}{2-p}\right)\left(\frac{2-p}{p}\right)\|T\|=c\|T\|>1$ by (3) in Theorem 1 and so $\left(\frac{c p}{2-p}\right) T \notin C_{p}$ for every $p$ with $0<p<1$.

(3) $\Rightarrow(1)$. If $\mathscr{A}$ is not commutative, then $w_{p}\left(\frac{c p}{2-p} T\right)=\frac{c\|T\|}{2-p}$ for some $T \in \mathscr{A}, T \neq 0$ and $T^{2}=0$. By choosing suitable $c$ and $p(\leqslant 2-c\|T\|)$ we may conclude that $\left(\frac{c p}{2-p}\right) T \in C_{p}$.

THEOREM 4 (Theorem $2[1]$ when $p=1$ ). The following statements are equivalent.

(1) $A$ is commutative.

(2) For any $p \geqslant 1, w_{p}(A+B) \leqslant 1+w_{p}(A B)$ for all self-adjoint operators $A$ and $B \in \mathscr{A}$ with $\|A\|=\|B\|=1$.

(3) For any $p$ with $0<p<1, w_{p}(A+B) \leqslant \frac{2-p}{p}+w_{p}(A B)$ for all self-adjoint operators $A$ and $B \in \mathscr{A}$ with $\|A\|=\|B\|=1$.

Proof. We shall adapt the original result in $[1] ; \mathscr{A}$ is commutative if and only if $\|A+B\| \leqslant 1+\|A B\|$ for all self-adjoint operators $A$ and $B \in \mathscr{A}$ with $\|A\|=\|B\|=1$.

(1) $\Rightarrow(2) . \quad w_{p}(A+B)=\|A+B\| \leqslant 1+\|A B\|=1+w_{p}(A B)$ by (3) in Theorem 1 .

(2) $\Rightarrow(1)$. If $\mathscr{A}$ is not commutative, then for some self-adjoint operators $A$ and $B$, $w_{p}(A+B)=\|A+B\|>1+\|A B\| \geqslant 1+w_{p}(A B)$ by (1) in Theorem 1 for the last inequality.

Theorem 1.

$(1) \Rightarrow(3) .\left(\frac{p}{2-p}\right) w_{p}(A+B)=\|A+B\| \leqslant 1+\|A B\|=1+\left(\frac{p}{2-p}\right) w_{p}(A B)$ by (3) in

(3) $\Rightarrow(1)$. If $\mathscr{A}$ is not commutative, for some self-adjoint operators $A$ and $B$,

$$
\left(\frac{p}{2-p}\right) w_{p}(A+B)=\|A+B\|>1+\|A B\| \geqslant 1+\left(\frac{p}{2-p}\right) w_{p}(A B)
$$

by (1) in Theorem 1 for the last inequality. 
We recall that $T \in \mathscr{A}$ satisfies the first order growth condition $\left(G_{1}\right)$ if $\left\|(u-T)^{-1}\right\|=$ $1 / d(u)$ for all $u \notin \sigma(T)$, the spectrum of $T$, where $d(u)$ denotes the distance from $u$ to $\sigma(T)$. It is known that $\left\|(u-T)^{-1}\right\| \geqslant 1 / d(u)$ holds for any $T \in \mathscr{A}$, and a normal operator satisfies condition $\left(G_{1}\right)$. We shall next generalize this to operator radii and prove the following result.

THEOREM 5. The following statements are equivalent.

(1) $A$ is commutative.

(2) For $p \geqslant 1, w_{p}\left((u-T)^{-1}\right)=1 / d(u)$ for every $T \in \mathscr{A}$ and $u \notin \sigma(T)$.

.(3) For $0<p<1, w_{p}\left((u-T)^{-1}\right)=\frac{2-p}{p d(u)}$ for every $T \in \mathscr{A}$ and $u \notin \sigma(T)$.

Proof. (1) $\Rightarrow(2)$. Since $(u-T)^{-1}$ is normal,

$$
d(u) w_{p}\left((u-T)^{-1}\right)=d(u)\left\|(u-T)^{-1}\right\|=d(u)(1 / d(u))=1
$$

by (3) in Theorem 1.

(2) $\Rightarrow(1)$. Let $T \in \mathscr{A}, T^{k}=0, k \geqslant 2$ and $T^{k-1} \neq 0$; then $\sigma(T)=\{0\}$. We shall show that if $T$ satisfies the condition $w_{p}\left((u-T)^{-1}\right) \leqslant 1 / d(u)$, that is $u(u-T)^{-1} \in C_{p}$ for any complex number $u \neq 0$, then $T=0$. To this end, let $z=1$ in the inequality $\left({ }^{*}\right)$. We obtain

$$
(p-2)\left(I-u(u-T)^{-1}\right)^{*}\left(I-u(u-T)^{-1}\right)+\left(I-u(u-T)^{-1}\right)+\left(I-u(u-T)^{-1}\right)^{*} \geqslant 0 .
$$

Let the left hand side in the above be $F$; then $(u-T)^{*} F(u-T) \geqslant 0$ and so

$$
(p-2) T^{*} T-(u-T)^{*} T-T^{*}(u-T) \geqslant 0 .
$$

We claim that $N(T) \subseteq N\left(T^{*}\right)$, where $N(\cdot)$ denotes the null space. Let $x \in N(T)$, and $Q$ be the left side in above inequality; then $Q(x)=-T^{*}(u x)$ so that $\left\|Q^{1 / 2}(x)\right\|^{2}=(Q(x), x)=$ $\left(-T^{*}(u x), x\right)=0$ and hence $T^{*}(x)=0$. Now, for any $x \in H, 0=T^{k}(x)=T^{*} T^{k-1}(x)$ by the claim. It follows that $T^{k-1}=0$, contrary to our assumption. Thus, $T=0$.

(1) $\Rightarrow(3)$. Normality of $(u-T)^{-1}$ implies that

$$
\frac{p d(u)}{2-p} w_{p}\left((u-T)^{-1}\right)=\frac{p d(u)}{2-p}\left\|(u-T)^{-1}\right\| \frac{2-p}{p}=1
$$

by (3) in Theorem 1 .

(3) $\Rightarrow(1)$. The proof may be carried out in a manner similar to the one above by showing that $T=0$. To make computation simple let $u=1$, and $z=-1$ in $\left(^{*}\right)$. Since $\frac{p}{2-p}(I-T)^{-1} \in C_{p}$, it follows that

$$
S=(p-2)\left(\frac{2}{2-p}-T\right)^{*}\left(\frac{2}{2-p}-T\right)+(I-T)^{*}\left(\frac{2}{2-p}-T\right)+\left(\frac{2}{2-p}-T\right) *(I-T) \geqslant 0 .
$$


Let $x \in N(T)$ and consider $S(x)$; after a few simplifications we have

$$
S(x)=\frac{p}{p-2} T^{*}(x) .
$$

The remainder of the proof is the same as above.

Finally, we remark that the above conditions on $T$ may be relaxed and the result still holds. For example, $u \in U \backslash \sigma(T)$, where the set $U$ need not contain $\sigma(T)$ but $U \backslash \sigma(T)$ must be non-empty.

\section{REFERENCES}

1. M. J. Crabb, J. Duncan and C. M. McGregor, Characterizations of commutativity for $C^{*}$-algebras, Glasgow Math. J. 15 (1974), 172-175.

2. J. Dixmier, $C^{*}$-algebras, (North-Holland, 1977).

3. J. A. R. Holbrook, On the power-bounded operators of Sz-Nagy and Foias, Acta Sci. Math., 29 (1968), 299-310.

4. B. Sz-Nagy and C. Foias, Harmonic analysis of operators on Hilbert Space, (Akadémiai Kiado, Budapest, 1970).

Department of Mathematics

BISHOP'S UNIVERSITY

LENNOXVILLE, QuebeC

CANADA 